\title{
The gene that hits the hot spot
}

Sites of meiotic recombination are highly localized on eukaryotic genomes, but the reason for this remains puzzling. Three papers now identify a histone methyltransferase as a key controller of crossover 'hot spots': a finding that has implications for the molecular control of recombination and its evolution.

A 5.4-Mb region of chromosome 17 is known to be responsible for differences in the distribution of recombination among mouse strains. In a new study, Baudat and colleagues reasoned that a likely controller of recombination is

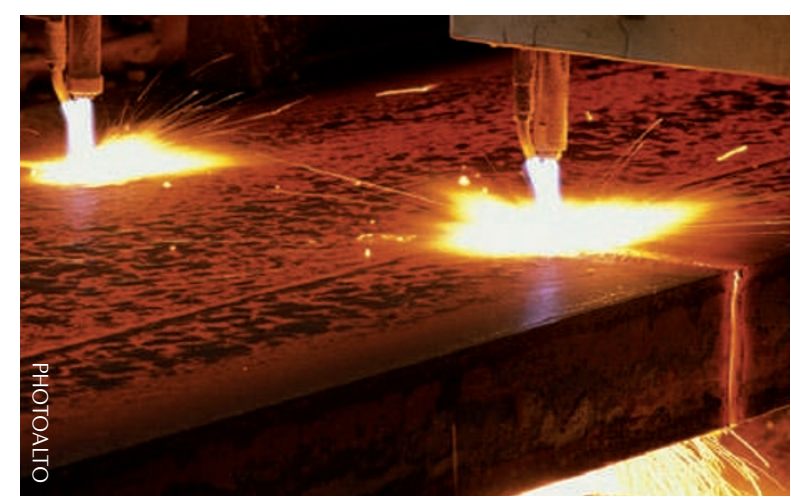

PR domain-containing 9 ( $\operatorname{Prdm} 9)$, which lies within this region and encodes a histone $\mathrm{H} 3$ lysine 4 (H3K4) methyltransferase; levels of $\mathrm{H} 3 \mathrm{~K} 4$ methylation correlate with hot spot activity, and PRDM9 contains DNA-binding zinc fingers. They confirmed their prediction by identifying polymorphisms in the zinc finger domain of $\operatorname{Prdm} 9$ and its human orthologue that are consistent with differing recombination distributions. Independently, Parvanov and colleagues refined the location of the hot spot regulator to a region containing four genes. Three of the genes lacked variation between strains with different recombination patterns, so the fourth - Prdm9 - had to be the regulator.

Myers and colleagues previously found a 13-bp motif that is associated with $\sim 40 \%$ of recombination hot spots in the human genome. In a new paper, the same group predict - using a computational search - that the zinc finger domain of PRDM9 binds this motif. Baudat and colleagues confirmed in vitro that PRDM9 specifically binds the hot spot motif.
Chimpanzees share almost no hot spot locations with humans, and by statistical analysis Myers et al. showed that the 13-bp motif does not recruit hot spots in chimpanzees. They found that chimpanzee PRDM9 has a dramatically different binding specificity; remarkably rapid evolution of PRDM9 provides a likely explanation for the lack of hot spot conservation between species.

Attention is now likely to focus on elucidating how PRDM9 functions. An H3K4 methyltransferase also affects hot spots in yeast, so a link between histone modification and recombination might prove to be a general eukaryotic phenomenon.

Mary Muers

ORIGINAL RESEARCH PAPERS Baudat, F. et al. PRDM9 is a major determinant of meiotic recombination hotspots in humans and mice. Science 31 Dec 2009 (doi:10.1126/ science.1183439) | Parvanov, E. D., Petkov, P. M. \& Paigen, K. Prdm9 controls activation of mammalian recombination hotspots. Science 31 Dec 2009 (doi:10.1126/science.1181495)| Myers, S. et al. Drive against hotspot motifs in primates implicates the PRDM9 gene in meiotic recombination. Science 31 Dec 2009 (doi:10.1126/science.1182363) 